\title{
Type 1 diabetes risk assessment: improvement by follow-up measurements in young islet autoantibody-positive relatives
}

\author{
P. Achenbach $\cdot$ K. Warncke $\cdot$ J. Reiter . \\ A. J. K. Williams • A. G. Ziegler • P. J. Bingley • \\ E. Bonifacio
}

Received: 25 April 2006 / Accepted: 31 July 2006 / Published online: 26 September 2006

(C) Springer-Verlag 2006

\begin{abstract}
Aims/hypothesis Combinations of autoantibody characteristics, including antibody number, titre, subclass and epitope have been shown to stratify type 1 diabetes risk in islet autoantibody-positive relatives. The aim of this study was to determine whether autoantibody characteristics change over time, the nature of such changes, and their implications for the development of diabetes.

Methods Five-hundred and thirteen follow-up samples from 141 islet autoantibody-positive first-degree relatives were tested for islet autoantibody titre, IgG subclass, and GAD and IA-2 antibody epitope. All samples were categorised according to four risk stratification models. Relatives had a median follow-up of 6.8 years and 48 developed diabetes during follow-up. Survival analysis was used to determine the probability of change in risk category and of progression to diabetes.

Results For each stratification model, the majority of relatives (71-81\%) remained in the same risk category throughout follow-up. In the remainder, changes occurred both from lower
\end{abstract}

P. Achenbach · K. Warncke J. Reiter · A. G. Ziegler $(\bowtie) \cdot$

E. Bonifacio

Diabetes Research Institute,

Koelner Platz 1,

80804 Munich, Germany

e-mail: anziegler@1rz.uni-muenchen.de

\section{A. J. K. Williams • P. J. Bingley}

Diabetes and Metabolism,

Department of Clinical Science at North Bristol,

University of Bristol,

Bristol, UK

E. Bonifacio

Istituto Scientifico San Raffaele,

Milan, Italy to higher and from higher to lower risk categories. For all four models, relatives aged $<15$ years were more likely to change risk category than those aged $>15$ years $(0.001<p<0.03)$. Relatives whose autoantibody status changed from low- to high-risk categories had a higher risk of diabetes than relatives who remained in low-risk categories, and inclusion of autoantibody status during follow-up improved diabetes risk stratification in Cox proportional hazards models $(p<0.001)$. Conclusions/interpretation Changes in islet autoantibodies are relevant to pathogenesis, and are likely to signal alterations in the disease process. Detection of changes through follow-up measurement will improve diabetes risk stratification, particularly in young individuals.

Keywords GAD $\cdot$ IA- $2 \cdot$ IA- $2 \beta \cdot$ Insulin autoantibody Islet autoantibody · Prediction · Type 1 diabetes

$\begin{array}{ll}\text { Abbreviations } \\ \text { BOX } & \text { Bart's-Oxford } \\ \text { IAA } & \text { autoantibodies to insulin } \\ \text { IA-2A } & \text { autoantibodies to IA-2 } \\ \text { IA-2 } \beta \text { A } & \text { autoantibodies to IA-2 } \beta \\ \text { GADA } & \text { autoantibodies to GAD } \\ \text { IQR } & \text { interquartile range }\end{array}$

\section{Introduction}

Accurate assessment of risk of type 1 diabetes in non-diabetic individuals is necessary to identify those suitable for recruitment into intervention trials aiming to prevent the clinical onset of diabetes [1]. Islet cell autoantibodies and autoantibodies to insulin (IAA), the $65-\mathrm{kDa}$ isoform of 
glutamate decarboxylase (GADA), and the protein tyrosine phosphatase-like antigen IA-2 (IA-2A) are established risk markers for type 1 diabetes $[2,3]$, and risk is related to the titre of islet cell antibodies $[4,5]$ and to the number of autoantibodies [6-10]. In addition, we recently reported that risk can be stratified using algorithms of autoantibody characteristics, including IAA and IA-2A titre, IAA and IA- $2 \mathrm{~A}$ IgG subclass, and antibody binding to the IA- $2 \beta$ autoantigen [11]. Applying models based on these algorithms to a single sample, diabetes risk in autoantibodypositive relatives could be further stratified from less than $10 \%$ to almost $100 \%$ within 5 years of screening. To use these stratification models most efficiently, we need to know whether an individual's assigned risk category changes over time, and the implications of any such changes for the development of diabetes. We therefore examined risk assignment based on these models in serial samples from 141 antibody-positive first-degree relatives, and assessed the effect of changes on diabetes risk.

\section{Subjects and methods}

\section{Subjects}

This study was performed on 141 of the 180 autoantibodypositive relatives from the Bart's-Oxford (BOX; $n=67$ ) and the Munich family studies $(n=74)[12,13]$ previously used to define the risk stratification models [11]. Relatives for this study were selected on the basis of the availability of followup samples to allow testing of all antibody characteristics (titre, IgG subclasses of IAA and IA-2A, antibodies to IA- $2 \beta$ [IA-2 $\beta A]$ ). The study cohort contained 49 offspring (30 sons, 19 daughters), 52 siblings ( 24 brothers, 28 sisters), and 40 parents (17 fathers, 23 mothers) of the diabetic proband. The first available autoantibody-positive serum sample and subsequent follow-up samples from these relatives were used for antibody characterisation (total 513 samples; median number of samples per relative 3, interquartile range (IQR) 2-4, range $2-11$; median time between samples 1.8 years, IQR 1.2 2.4 years). The median age of relatives at time of first sample was 15.0 years (IQR 8.3-32.5 years). Relatives were prospectively monitored for the development of diabetes over a median follow-up period of 6.8 years (IQR 4.6-12.3 years) for a total of 1145 subject-years. The median follow-up was 5.9 years (IQR 3.7-8.2) for relatives aged $<15$ years, and 9.7 years (IQR 5.8-13.8) for relatives aged $>15$ years $(p<$ 0.001 ). Of the 141 autoantibody-positive relatives, 48 developed diabetes during follow-up (median time to diabetes 4.4 years, IQR 2.2-6.3 years). Diabetes was diagnosed using World Health Organization/American Diabetes Association criteria [14]. The respective local ethical committees approved the BOX and Munich family studies. All relatives in this study gave informed consent, and the study was carried out in accordance with the Declaration of Helsinki as revised in 2000 http://www.wma.net/e/policy/b3.htm, last accessed in August 2006).

Islet autoantibody measurements

IAA, GADA and IA-2A were measured in all 513 samples by protein $\mathrm{A} / \mathrm{G}$ radiobinding assays, as previously described [9, 15], using $\mathrm{Tyr}^{14} \mathrm{~A}{ }^{125}$ I-labelled human insulin (Aventis, Frankfurt, Germany), and $\left.{ }^{35} \mathrm{~S}\right]$ methionine-labelled, in vitrotranslated recombinant human GAD65 and IA-2, respectively. IAA- and/or IA-2A-positive samples with antibody titres above the discriminatory range of the assays were titrated [11]. The thresholds for positivity in each assay corresponded to the 99th percentile for antibody titre in control subjects. These assays had sensitivities and specificities of $70 \%$ and $99 \%$ (IAA), $86 \%$ and $93 \%$ (GADA), and $72 \%$ and $100 \%$ (IA-2A), respectively, in the Third Diabetes Autoantibody Standardization Program workshop.

IA-2 $\beta$ A were determined in IA-2A-positive samples by protein $\mathrm{A} / \mathrm{G}$ radiobinding assays using a $\left[{ }^{35} \mathrm{~S}\right]$ methioninelabelled, in vitro-translated recombinant human intracellular portion of IA-2 $\beta$ (amino acids 662-1033) $[11,16]$. IgG subclasses of IAA and IA-2A were determined by radiobinding assays as previously described $[11,17]$.

Data analysis

Diabetes risk stratification models All 513 samples were classified according to categories of four diabetes risk stratification models, which had been previously developed on the basis of autoantibody status in the first available islet autoantibody-positive samples from 180 relatives [11]. Models are described in Fig. 1.

Statistical analysis The Mann-Whitney $U$-test or KruskalWallis $H$-test was used to compare differences in age between groups. The Wilcoxon test was used to compare directional changes in model risk categories between the first and last sample. Life table analysis was used to determine the probability of change in model category and the diabetes risk. For analysis of the probability of change in model category, the time to event was defined by the first autoantibody-positive sample, and the end of follow-up was defined by the sample in which first change in category occurred or the date of the last sample tested in relatives who did not change category. The probability of change in category was compared between relatives $<15$ and $\geq 15$ years of age (50th percentile for age) using the log rank test. For analysis of diabetes risk, the time to event was defined from the first change in model category or from the first autoantibody-positive sample in relatives who did not 


\section{Model 1}

Stratification based on:

Number of islet autoantibodies

(IAA, IA-2A, GADA)

Category 1

One autoantibody

\section{Model 2}

Stratification based on:

High titre of

IAA (>3rd quartile)

and IA-2A (>1st quartile)
Model 3

Stratification based on:

High-risk characteristics:

High titre IA-2A (>1st quartile)

and IgG2 or IgG4 IA-2A

and $\lg G 2$, IgG3 or IgG4 IAA

\section{Model 4}

Stratification based on:

Status of

IA-2A and IA-2 $\beta A$

\section{Category 1 \\ No high-risk \\ characteristics}

Neither IAA nor IA-2A

at high titre

Category 2

Any two autoantibodies

Category 3

All three autoantibodies
Category 2

One of IAA or IA-2A at high titre

Category 3

Both of IAA and IA-2A at high titre
One high-risk
Category 2 characteristic

\section{Category 3 \\ Any two high-risk characteristics}

Category 4

All three high-risk characteristics
Category 1

IA-2A-negative

Category 2

IA-2A-positive and

IA-2ßA-negative

\section{Category 3}

IA-2ßA-positive
Fig. 1 Type 1 diabetes risk stratification models based on islet autoantibody characteristics. Shaded model categories represent characteristics that stratified 10-year diabetes risk above $50 \%$ in the first available autoantibody-positive screening samples from 180 first-degree relatives [11]. Low-risk categories are Category 1 of Models 1 and 2, and Categories 1 and 2 of Models 3 and 4 (all with a priori associated 10-year risks $<50 \%$ ). High-risk categories are Categories 2 and 3 of Models 1 and 2, Categories 3 and 4 of Model 3, and Category 3 of Model 4 (all with a priori associated 10-year risks $>50 \%$ ). In Model 2, IAA $\geq 9$ units were considered to be high titre (titre above the 75th percentile of cohort). In Models 2 and 3, IA-2A $\geq 27$ units (approximately 50 WHO units, titre above the 25th percentile of cohort) were considered to be high titre change category, and the end of follow-up was defined as date of diagnosis, date of entry into an intervention trial or date of last contact. Survival between groups was compared using the log rank test. For all analyses, a two-tailed $p$ value of 0.05 was considered significant; values were not corrected for number of comparisons using the four models. Cox proportional hazards models were used to analyse the influence of change over time on the performance of each model. The outcome was diagnosis of diabetes, and assigned risk categories were entered as time-dependent covariates with 1-year intervals. The category assigned to each time interval was the autoantibody status in the last sample prior to that time point. Improvement in risk stratification using models based on first measurement and follow-up samples was assessed by change in log likelihood ratio. All statistical analyses were performed using the Statistical Package for Social Science, version 13.0 (SPSS, Chicago, IL, USA).

\section{Results}

Classification according to risk categories of each of the four stratification models was done for 513 follow-up samples of
141 relatives. Example timelines are shown for Models 1 and 4 (Fig. 2). For each model, the majority of relatives remained in the same risk category throughout follow-up $(71 \%$ of relatives in Model 1, 75\% in Model 2, 72\% in Model 3, 81\% in Model 4). In relatives whose risk category changed, individual changes were usually unidirectional rather than fluctuating (Table 1). A preferential direction of change was observed only for Model 4 (Fig. 2b), in which shifts from lower to higher risk categories were more frequent than shifts from higher to lower risk categories $(p=0.03$; not significant for Models 1, 2 or 3$)$. Three relatives (2.1\%) lost all antibody reactivity on follow-up (age at first sample 16, 20 and 47 years). None of these relatives re-developed islet autoantibodies and none developed diabetes.

Relatives who changed risk category were younger at time of first autoantibody-positive sample than relatives whose category remained stable (Table 1 ). The 5-year probabilities of any change in category in relatives who were $<15$ years of age compared with older relatives were $44 \%(95 \% \mathrm{CI}$ $30.3-57.7)$ vs 17\% (95\% CI 7.2-26.8; $p=0.001)$ for Model 1, 28\% (95\% CI 16.2-39.8) vs 16\% (95\% CI 6.2-25.8; $p=$ 0.01 ) for Model 2, 37\% (95\% CI 23.3-50.7) vs 24\% (95\% CI 12.2-35.8; $p=0.03$ ) for Model 3 , and 25\% (95\% CI 13.2$36.8)$ vs $12 \%(95 \%$ CI 2.2-21.8; $p=0.008)$ for Model 4. 
Fig. 2 Classification of 141 antibody-positive relatives over time into type 1 diabetes risk categories using stratification Model 1, based on islet autoantibody number (a), and Model 4, based on autoantibodies to IA-2 and IA-2 $\beta$ (b). The 513 samples from 141 relatives were categorised according to model risk categories (ordinate) and plotted against age (abscissa). Follow-up samples from individuals are connected by lines. Lines are differently coloured for relatives who did not change in risk category (black), those who changed to a higher risk category (red), those who changed to a lower risk category (yellow), and those who fluctuated in risk category (blue)

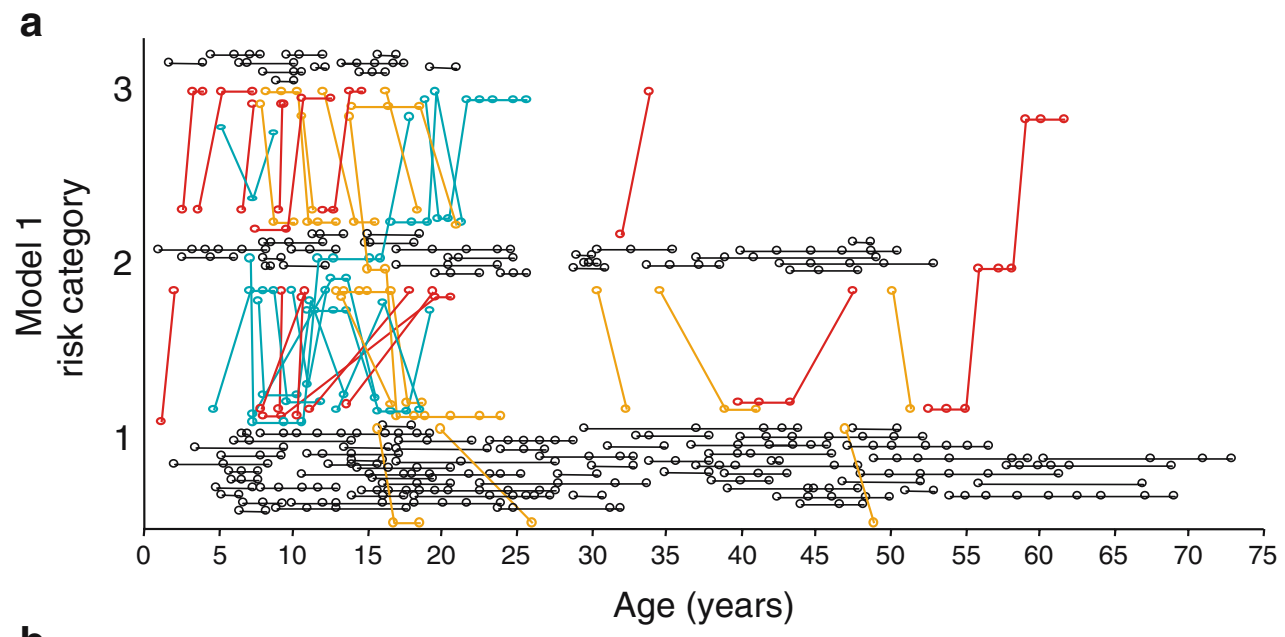

b

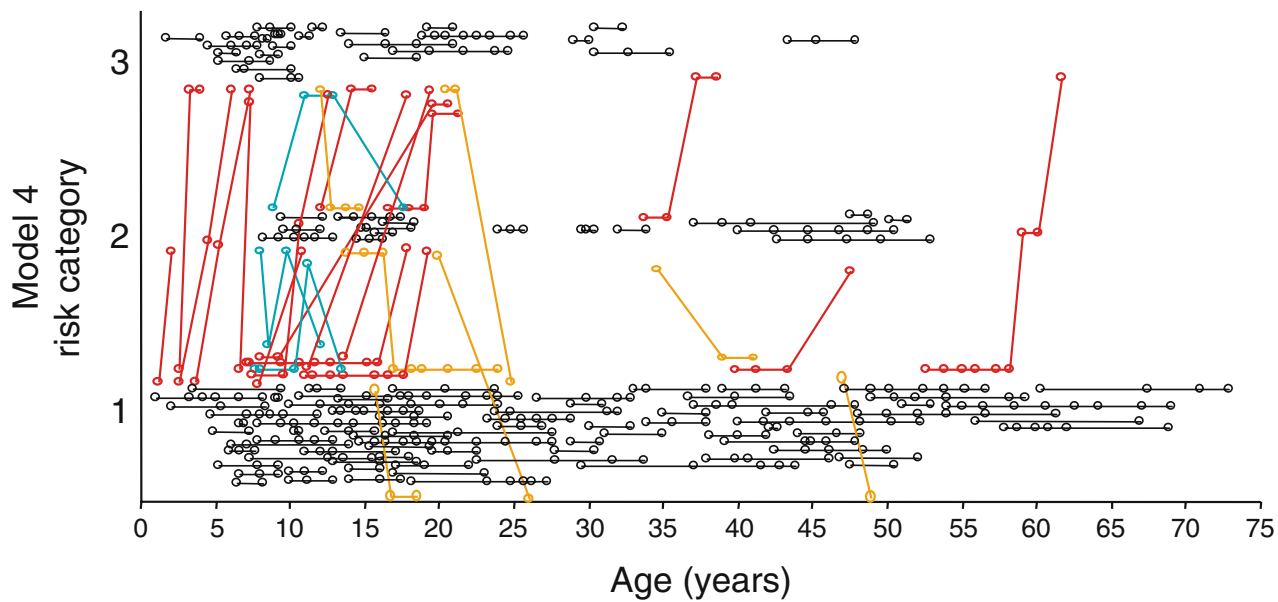

These associations remained significant after adjustment for the number of samples per subject and median time between sample collections. Younger age was also associated with higher risk categories ( $p<0.05$ for each model).

The overall 5-year and 10-year risks of diabetes in the 141 relatives were $20 \%(95 \%$ CI $13.3-26.7)$ and $36 \%$
(27.1-44.9), respectively. The results of life table analysis with respect to change or stability of model categories over time are shown in Fig. 3. Changes from low-risk to highrisk categories identified groups of relatives at higher diabetes risk than those who remained stable in low-risk categories $(p=0.02$, Model 1; $p<0.001$, Models 2, 3 and 4;

Table 1 Age at time of first sample in relation to stability or change in model risk categories on follow-up

\begin{tabular}{|c|c|c|c|c|c|c|c|c|}
\hline & \multicolumn{2}{|c|}{ Model 1} & \multicolumn{2}{|c|}{ Model 2} & \multicolumn{2}{|c|}{ Model 3} & \multicolumn{2}{|c|}{ Model 4} \\
\hline & $n$ & $\begin{array}{l}\text { Median age, years } \\
\text { (IQR) }\end{array}$ & $n$ & $\begin{array}{l}\text { Median age, years } \\
\text { (IQR) }\end{array}$ & $n$ & $\begin{array}{l}\text { Median age, years } \\
\text { (IQR) }\end{array}$ & $n$ & $\begin{array}{l}\text { Median age, years } \\
\text { (IQR) }\end{array}$ \\
\hline $\begin{array}{l}\text { Relatives who remained } \\
\text { stable in risk category }\end{array}$ & 100 & $17.1(9.6-36.9)^{\mathrm{a}}$ & 106 & $16.8(9.4-34.7)^{\mathrm{a}}$ & 102 & $17.0(9.9-35.4)^{\mathrm{a}}$ & 114 & $16.2(9.4-34.3)^{\mathrm{a}}$ \\
\hline $\begin{array}{l}\text { Relatives who changed risk } \\
\text { category }\end{array}$ & 41 & $11.2(7.8-16.5)^{\mathrm{a}}$ & 35 & $12.1(7.5-19.3)^{\mathrm{a}}$ & 39 & $12.1(6.7-19.3)^{\mathrm{a}}$ & 27 & $11.2(7.5-20.0)^{\mathrm{a}}$ \\
\hline Up & 16 & $9.1(6.9-13.3)^{\mathrm{b}}$ & 18 & $10.6(5.7-15.9)$ & 18 & $11.7(4.3-17.1)$ & 17 & $8.0(5.2-15.2)^{\mathrm{b}}$ \\
\hline Down & 15 & $14.0(12.1-30.5)^{\mathrm{b}}$ & 11 & $13.8(8.3-20.0)$ & 13 & $12.0(7.3-18.3)$ & 7 & $20.0(13.8-34.6)^{\mathrm{b}}$ \\
\hline Fluctuating & 10 & $8.9(6.7-13.8)$ & 6 & $15.9(9.2-39.4)$ & 8 & $16.6(8.2-37.6)$ & 3 & $8.1(7.7-9.0)$ \\
\hline
\end{tabular}

${ }^{\text {a }}$ Difference in age between relatives who remained stable and relatives who changed in risk category; $p=0.007$, Model $1 ; p=0.02$, Models 2 and $3 ; p=0.03$, Model 4

${ }^{\mathrm{b}}$ Difference in age between relatives who changed up and relatives who changed down in risk category; $p=0.02$, Models 1 and 4 ; not significant, Models 2 and 3 
a

Model 1

Model 2

Model 3

Model 4
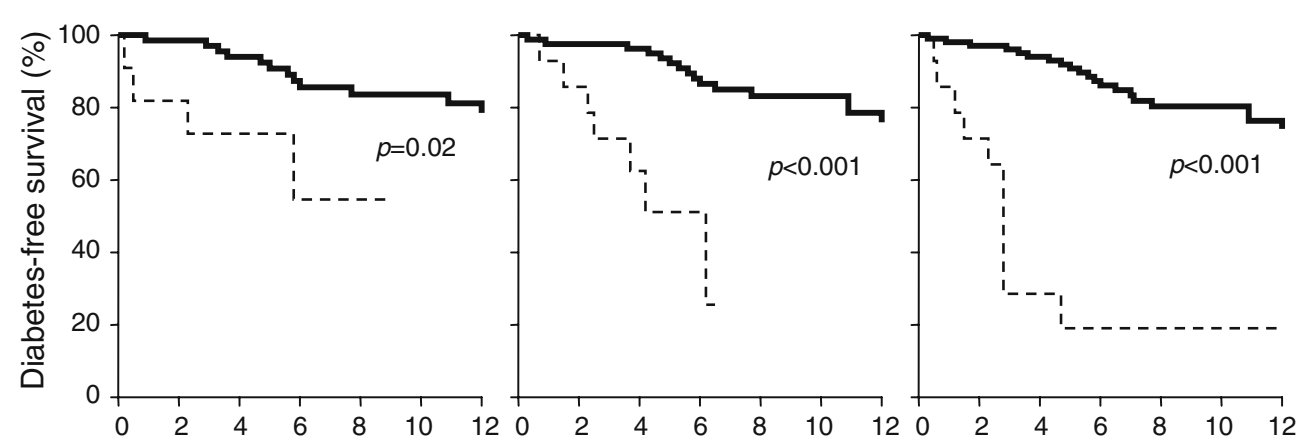

Follow-up (years)

b

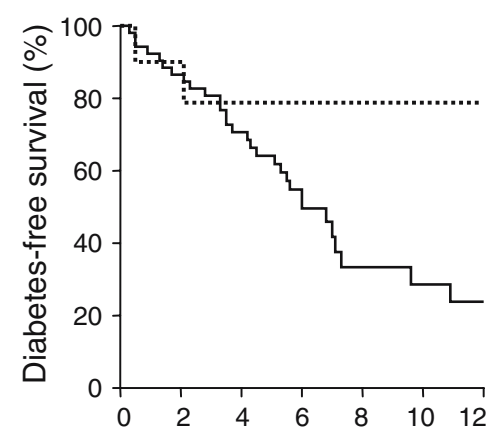

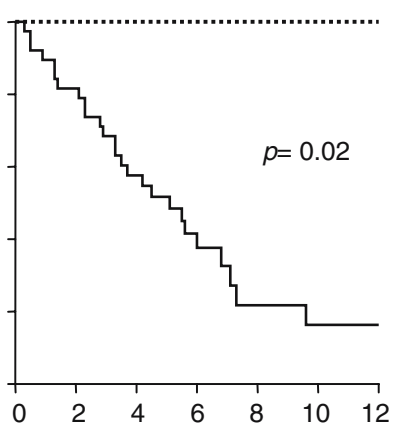
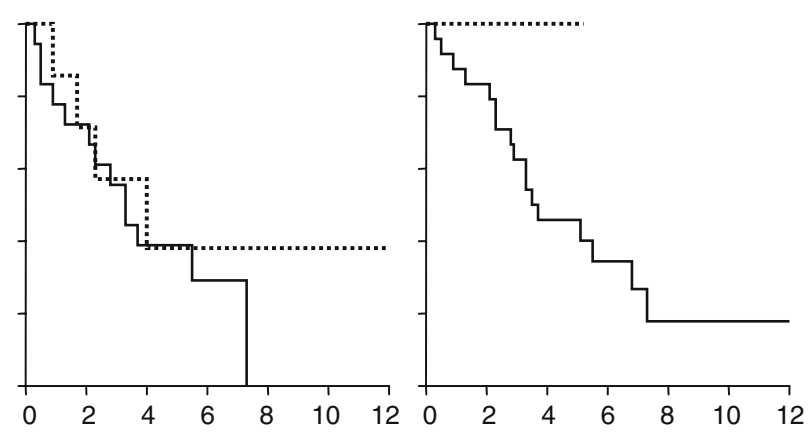

Follow-up (years)

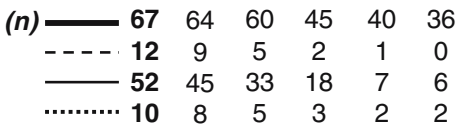

Fig. 3 Type 1 diabetes risk stratified in 141 antibody-positive relatives taking into consideration changes in risk category of stratification models on follow-up. Relatives were followed for diabetes development from time of first change in model risk category or first antibody-positive sample in relatives who did not change risk category throughout follow-up. For each model, relatives who

Fig. 3a). The risk of diabetes in relatives who changed to high-risk categories was similar to the risk in those who remained stable in high-risk categories throughout followup. In all models, few relatives changed from high-risk to low-risk categories, and only in Model 2 were relatives in this group at significantly lower risk than those who remained stable in high-risk categories $(p=0.02)$ (Fig. 3b). In Model 3, however, diabetes risk remained high in relatives who decreased from high- to low-risk categories.

Table 2 shows Cox proportional hazards models based on the antibody characteristics in the first sample alone (Cox Model A) and on the characteristics in follow-up samples (Cox Model B). Consideration of changes in categories over time significantly improved diabetes risk stratification $(p=$ 0.02 , Model 1; $p<0.001$, Models 2, 3, and 4; Table 2). Seventy-four relatives were in high-risk categories at screening or during follow-up using Model 1, 61 using

$\begin{array}{cccccccccccc}101 & 96 & 89 & 66 & 49 & 43 & \mathbf{1 0 2} & 95 & 90 & 66 & 49 & 44 \\ 15 & 10 & 4 & 1 & 1 & 1 & \mathbf{1 3} & 7 & 2 & 1 & 1 & 1 \\ 18 & 13 & 6 & 2 & 0 & 0 & \mathbf{2 4} & 20 & 10 & 5 & 2 & 2 \\ 7 & 5 & 2 & 2 & 2 & 1 & \mathbf{2} & 1 & 1 & 0 & 0 & 0\end{array}$

remained stable in low-risk categories (thick solid line) are compared with relatives who increased from low-risk to high-risk categories (broken line) (a), and relatives who remained stable in high-risk categories (thin solid line) are compared with relatives who decreased from high-risk to low-risk categories (dotted/dashed line) (b). Lowrisk and high-risk categories of models were considered as described

Model 2, 40 using Model 3, and 39 using Model 4. The 5year risk for progression to diabetes in these relatives was $33 \%$ in Model 1 (95\% CI 21.2-44.8), 45\% (95\% CI 31.358.7) in Model 2, 63\% (95\% CI 47.3-78.7) in Model 3, and $64 \%$ (95\% CI 48.3-79.7) in Model 4. Consideration of follow-up measurements increased sensitivity of the highrisk categories for identifying pre-type 1 diabetes from $67 \%$ to $75 \%(36 / 48)$ in Model 1, 54\% to 69\% (33/48) in Model 2, $35 \%$ to $58 \%$ (28/48) in Model 3, and 35\% to 54\% (26/48) in Model 4.

\section{Discussion}

We have shown that repeated testing of islet autoantibodies over time in autoantibody-positive first-degree relatives identified a subgroup of around $25 \%$ in whom there is a 
Table 2 Improvement of diabetes risk stratification models by considering changes in risk categories over time in 141 antibody-positive relatives

\begin{tabular}{|c|c|c|c|c|c|c|c|c|}
\hline \multirow[t]{2}{*}{ Variables in Cox's proportional hazards model } & \multicolumn{2}{|l|}{ Model 1} & \multicolumn{2}{|l|}{ Model 2} & \multicolumn{2}{|l|}{ Model 3} & \multicolumn{2}{|l|}{ Model 4} \\
\hline & $\begin{array}{l}\text { HR } \\
(95 \% \mathrm{CI})\end{array}$ & $p$ value & $\begin{array}{l}\text { HR } \\
(95 \% \mathrm{CI})\end{array}$ & $p$ value & $\begin{array}{l}\text { HR } \\
(95 \% \mathrm{CI})\end{array}$ & $p$ value & $\begin{array}{l}\text { HR } \\
(95 \% \mathrm{CI})\end{array}$ & $p$ value \\
\hline \multicolumn{9}{|l|}{ Cox Model A } \\
\hline Risk categories at first sample & & $<0.001$ & & $<0.001$ & & $<0.001$ & & $<0.001$ \\
\hline Category 1 & $1^{\mathrm{a}}$ & & $1^{\mathrm{a}}$ & & $1^{\mathrm{a}}$ & & $1^{\mathrm{a}}$ & \\
\hline Category 2 & $\begin{array}{l}3.9 \\
(2.0-7.5)\end{array}$ & $<0.001$ & $\begin{array}{l}3.4 \\
(1.8-6.2)\end{array}$ & $<0.001$ & $\begin{array}{l}2.3 \\
(1.1-4.7)\end{array}$ & 0.03 & $\begin{array}{l}2.5 \\
(1.2-5.2)\end{array}$ & 0.01 \\
\hline Category 3 & $\begin{array}{l}4.9 \\
(2.2-10.6)\end{array}$ & $<0.001$ & $\begin{array}{l}9.5 \\
(3.7-24.2)\end{array}$ & $<0.001$ & $\begin{array}{l}5.4 \\
(2.6-11.3)\end{array}$ & $<0.001$ & $\begin{array}{l}6.0 \\
(3.1-11.7)\end{array}$ & $<0.001$ \\
\hline Category 4 & & & & & $\begin{array}{l}19.4 \\
(6.7-56.1)\end{array}$ & $<0.001$ & & \\
\hline \multicolumn{9}{|l|}{ Cox Model B } \\
\hline $\begin{array}{l}\text { Risk categories as } \\
\text { time-dependent covariate }\end{array}$ & & $<0.001$ & & $<0.001$ & & $<0.001$ & & $<0.001$ \\
\hline Category 1 & $1^{\mathrm{a}}$ & & $1^{\mathrm{a}}$ & & $1^{\mathrm{a}}$ & & $1^{\mathrm{a}}$ & \\
\hline Category 2 & $\begin{array}{l}4.4 \\
(2.2-8.7)\end{array}$ & $<0.001$ & $\begin{array}{l}6.4 \\
(3.3-12.3)\end{array}$ & $<0.001$ & $\begin{array}{l}2.3 \\
(1.0-5.5)\end{array}$ & 0.06 & $\begin{array}{l}3.1 \\
(1.3-7.4)\end{array}$ & 0.01 \\
\hline Category 3 & $\begin{array}{l}7.0 \\
(3.2-15.2)\end{array}$ & $<0.001$ & $\begin{array}{l}17.4 \\
(6.9-44.3)\end{array}$ & $<0.001$ & $\begin{array}{l}11.2 \\
(5.7-21.9)\end{array}$ & $<0.001$ & $\begin{array}{l}10.6 \\
(5.5-20.5)\end{array}$ & $<0.001$ \\
\hline Category 4 & & & & & $\begin{array}{l}15.1 \\
(4.2-55.2)\end{array}$ & $<0.001$ & & \\
\hline $\begin{array}{l}\text { Improvement from } \\
\text { Model A to Model B }\end{array}$ & & 0.02 & & $<0.001$ & & $<0.001$ & & $<0.001$ \\
\hline
\end{tabular}

${ }^{\text {a }}$ Reference cell used in Cox's proportional hazards model. HR, hazard ratio

change in antibody status that is indicative of a different diabetes risk. Change in antibody status was usually persistent and occurred more often in children and adolescents than in adults. The findings are relevant to staging of diabetes risk and suggest that monitoring islet autoantibody status and risk categorised on the basis of antibody characteristics is likely to improve our ability to predict diabetes in relatives at risk.

The study was performed on autoantibody-positive relatives that have had up to 18 years of follow-up with frequent sampling. The cohort included 48 relatives who developed diabetes, as well as 47 who remained nondiabetic for more than 10 years. In this study we focused on the selected number of antibody characteristics, which we have previously found to be the most significant predictors of diabetes in the same cohort [11]. These characteristics allowed stratification of diabetes risk into three or more discrete model risk categories which, as shown by the data, were relatively robust and stable over time. Although changes in autoantibody titre, IgG subclass and epitope were observed in many relatives (data not shown), these did not usually result in changes in risk category. For example, changes in antibody characteristics of GADA did not affect risk categories in any of our models, and changes in antibody titre of IAA or IA-2A were not always associated with a change in risk category in Model 2 or 3 (i.e. titre change did not cross the 75th percentile for titre of IAA or the 25th percentile for titre of IA-2A). A limitation of the study is the fact that we did not assess the metabolic makers that have been shown to provide added specificity for disease progression in autoantibody-positive relatives [1820]. Similarly, we were unable to assess $T$ cell reactivity, which might have provided further insights into the relevance of our findings to disease pathogenesis [21].

Measures that monitor progression to type 1 diabetes should be sensitive in identifying changes relevant to pathogenesis, and changes in risk category should not be seen in the absence of relevant pathogenic events. Using standard (antibody number) and more sophisticated models of risk stratification based on islet autoantibodies, we report that risk categories were stable over time for the majority of relatives, and that the rate of progression to diabetes was reasonably linear in relatives in whom autoantibody status remained unchanged. Thus, for the majority of autoantibody-positive relatives, the risk assigned at screening provided a relatively accurate assessment throughout follow-up. These findings are in line with the remarkable concordance between the diabetes risk predicted from the screening samples and the actual diabetes progression in the European Nicotinamide Diabetes Intervention Trial (ENDIT) [22] and the Diabetes Prevention Trial-1 (DPT-1) [23, 24]. 
Follow-up measurement was useful in a minority of autoantibody-positive relatives, and the use of autoantibody status as a time-dependent covariate in the cohort significantly improved diabetes risk assessment in comparison with the use of the screening value alone. Around $20 \%$ of the relatives had a persistent change in antibody status. These were generally younger relatives, and few changes in antibody risk category were observed in adulthood. Changes were predominantly unidirectional in any single individual, but occurred in both directions within a given model (increase in risk category in some relatives and decrease in others). It is interesting that changes from low- to very-highrisk categories often occurred abruptly over a short time (Fig. 2b), suggesting that such changes mark pathogenic events that alter the course of the disease. This is also reflected by the observation that changes in autoantibody status were associated with changes in diabetes risk. In particular, changes to higher risk categories were associated with faster progression to diabetes. The effect of change in autoantibody status to lower risk categories was less consistently associated with change in actual risk. The number of relatives who changed from a high-risk to a lower risk category during follow-up was, however, small (two relatives in Model 4 to ten relatives in Model 1). A few of the relatives whose high-risk category fell actually progressed rapidly to diabetes. This phenomenon was generally seen in association with the loss or decrease of IAA titre during follow-up, and is consistent with previous reports that IAA titre is inversely related to age [25] and a loss of IAA may occur prior to diabetes onset [26].

The findings from this and previous studies [7, 27-29] indicate that age is a determinant of risk of type 1 diabetes. Younger islet autoantibody-positive relatives were more likely to be in high-risk categories and were more likely to change from low-risk to high-risk categories than older relatives. The majority of adult autoantibody-positive relatives had low-risk antibody profiles (e.g. single GADA-positive only). These relatives had a steady but slow rate of progression to diabetes. The measurement of additional markers, such as autoantibody affinity or metabolic testing, may better identify progressors in this subgroup [29-31].

We have shown that diabetes risk stratification using autoantibodies can be improved by considering criteria other than just antibody number $[6,7,10]$. Previously, algorithms that considered IAA and IA-2A titre, IgG subclass and/or IA-2 $\beta$ A status were able to stratify type 1 diabetes risk at a single time point better than classification on the basis of antibody number alone [11]. Here, we find that these same algorithms improve risk stratification even further when autoantibody characteristics in follow-up samples are also considered. Model 4, based simply on IA- $2 \beta$ A positivity was particularly powerful in identifying relatives at high risk. A total of 39 relatives had IA-2 $\beta$ A at screening or during follow-up. This group had an overall 5 -year risk of diabetes of $64 \%$, and included 26 of the 48 relatives who developed diabetes during follow-up. Of the four models described, Model 1, based on antibody number, and Model 4, based on autoantibodies to IA-2 and IA-2 $\beta$, are likely to be the most practical for general application, and measurement of IA-2 $\beta$ A could be introduced to large-scale screening and recruitment to intervention trials.

In summary, our results support the use of follow-up measurements of islet autoantibodies in autoantibodypositive relatives for improved diabetes risk stratification and increased sensitivity of identification of pre-type 1 diabetes. Determination of antibody characteristics in follow-up samples identified up to $50 \%$ more relatives who had a $>50 \%$ risk of diabetes. Changes were mainly seen in the younger relatives, suggesting that follow-up autoantibody testing could be restricted to children and adolescents.

Acknowledgements This work was supported by a grant from the Deutsche Forschungsgemeinschaft (German Research Foundation) (ZI 310/12-6). The BOX study is supported by Diabetes UK. P. Achenbach received support from the Juvenile Diabetes Research Foundation (11-2005-1117). The authors thank A. Knopff, U. Mollenhauer, K. Koczwara and A. Norcross for technical support, and M. Walter and M. Hummel for clinical assistance. This work forms part of the dissertations of K. Warncke and J. Reiter.

\section{References}

1. Achenbach P, Bonifacio E, Ziegler AG (2005) Predicting type 1 diabetes. Curr Diab Rep 5:98-103

2. Atkinson MA, Eisenbarth GS (2001) Type 1 diabetes: new perspectives on disease pathogenesis and treatment. Lancet 358:221-229

3. Achenbach P, Bonifacio E, Koczwara K, Ziegler AG (2005) Natural history of type 1 diabetes. Diabetes 54:S25-S31

4. Bonifacio E, Bingley PJ, Shattock M et al (1990) Quantification of islet-cell antibodies and prediction of insulin-dependent diabetes. Lancet 335:147-149

5. Bingley PJ (1996) Interactions of age, islet cell antibodies, insulin autoantibodies, and first-phase insulin response in predicting risk of progression to IDDM in ICA+ relatives: the ICARUS data set. Islet cell antibody register users study. Diabetes 45:1720-1728

6. Bingley PJ, Christie MR, Bonifacio E et al (1994) Combined analysis of autoantibodies improves prediction of IDDM in islet cell antibody-positive relatives. Diabetes 43:1304-1310

7. Verge CF, Gianani R, Kawasaki E et al (1996) Prediction of type I diabetes in first-degree relatives using a combination of insulin, GAD, and ICA512bdc/IA-2 autoantibodies. Diabetes 45:926-933

8. Kulmala P, Savola K, Petersen JS et al (1998) Prediction of insulin-dependent diabetes mellitus in siblings of children with diabetes. A population-based study. The Childhood Diabetes in Finland Study Group. J Clin Invest 101:327-336

9. Ziegler AG, Hummel M, Schenker M, Bonifacio E (1999) Autoantibody appearance and risk for development of childhood 
diabetes in offspring of parents with type 1 diabetes: the 2-year analysis of the German BABYDIAB Study. Diabetes 48:460-468

10. Krischer JP, Cuthbertson DD, Yu L et al (2003) Screening strategies for the identification of multiple antibody-positive relatives of individuals with type 1 diabetes. J Clin Endocrinol Metab 88:103-108

11. Achenbach P, Warncke K, Reiter J et al (2004) Stratification of type 1 diabetes risk on the basis of islet autoantibody characteristics. Diabetes 53:384-392

12. Gardner SG, Gale EA, Williams AJ et al (1999) Progression to diabetes in relatives with islet autoantibodies. Is it inevitable? Diabetes Care 22:2049-2054

13. Dittler J, Seidel D, Schenker M, Ziegler AG (1998) GADIA2combi determination as first-line screening for improved prediction of type 1 diabetes in relatives. Diabetes 47:592-597

14. American Diabetes Association (1997) Report of the Expert Committee on the Diagnosis and Classification of Diabetes Mellitus. Diabetes Care 20:1183-1197

15. Naserke HE, Bonifacio E, Ziegler AG (1999) Immunoglobulin G insulin autoantibodies in BABYDIAB offspring appear postnatally: sensitive early detection using a protein $\mathrm{A} / \mathrm{G}$-based radiobinding assay. J Clin Endocrinol Metab 84:1239-1243

16. Bonifacio E, Lampasona V, Bingley PJ (1998) IA-2 (islet cell antigen 512) is the primary target of humoral autoimmunity against type 1 diabetes-associated tyrosine phosphatase autoantigens. J Immunol 161:2648-2654

17. Bonifacio E, Scirpoli M, Kredel K, Fuchtenbusch M, Ziegler AG (1999) Early autoantibody responses in prediabetes are IgG1 dominated and suggest antigen-specific regulation. J Immunol 163:525-532

18. Chase HP, Cuthbertson DD, Dolan LM et al (2001) First-phase insulin release during the intravenous glucose tolerance test as a risk factor for type 1 diabetes. J Pediatr 138:244-249

19. Keskinen P, Korhonen S, Kupila A et al (2002) First-phase insulin response in young healthy children at genetic and immunological risk for Type I diabetes. Diabetologia 45:1639-1648

20. Fourlanos S, Narendran P, Byrnes GB, Colman PG, Harrison LC (2004) Insulin resistance is a risk factor for progression to type 1 diabetes. Diabetologia 47:1661-1667
21. Arif S, Tree TI, Astill TP et al (2004) Autoreactive T cell responses show proinflammatory polarization in diabetes but a regulatory phenotype in health. J Clin Invest 113:451-463

22. Gale EA, Bingley PJ, Emmett CL, Collier T (2004) European Nicotinamide Diabetes Intervention Trial (ENDIT): a randomised controlled trial of intervention before the onset of type 1 diabetes. Lancet 363:925-931

23. Diabetes Prevention Trial-Type 1 Diabetes Study Group (2002) Effects of insulin in relatives of patients with type 1 diabetes mellitus. N Engl J Med 346:1685-1691

24. Skyler JS, Krischer JP, Wolfsdorf J et al (2005) Effects of oral insulin in relatives of patients with type 1 diabetes: The Diabetes Prevention Trial - Type 1. Diabetes Care 28:1068-1076

25. Vardi P, Ziegler AG, Mathews JH et al (1988) Concentration of insulin autoantibodies at onset of type I diabetes. Inverse loglinear correlation with age. Diabetes Care 11:736-739

26. Achenbach P, Ziegler AG (2005) Diabetes-related antibodies in euglycemic subjects. Best Pract Res Clin Endocrinol Metab 19:101-117

27. Bingley PJ, Williams AJ, Gale EA (1999) Optimized autoantibody-based risk assessment in family members. Implications for future intervention trials. Diabetes Care 22:1796-1801

28. Hummel M, Bonifacio E, Schmid S, Walter M, Knopff A, Ziegler AG (2004) Brief communication: early appearance of islet autoantibodies predicts childhood type 1 diabetes in offspring of diabetic parents. Ann Intern Med 140:882-886

29. Bingley PJ, Gale EA; The European Nicotinamide Diabetes Intervention Trial (ENDIT) Group (2006) Progression to type 1 diabetes in islet cell antibody-positive relatives in the European Nicotinamide Diabetes Intervention Trial: the role of additional immune, genetic and metabolic markers of risk. Diabetologia 49:881-890

30. Achenbach P, Koczwara K, Knopff A, Naserke H, Ziegler AG, Bonifacio E (2004) Mature high-affinity immune responses to (pro)insulin anticipate the autoimmune cascade that leads to type 1 diabetes. J Clin Invest 114:589-597

31. Mrena S, Virtanen SM, Laippala P et al (2006) Models for predicting type 1 diabetes in siblings of affected children. Diabetes Care 29:662-667 